\title{
Dietary Supplementation of Gamma-Linolenic Acid Improves Skin Parameters in Subjects with Dry Skin and Mild Atopic Dermatitis
}

\author{
Asuka Kawamura $^{1 *}$, Katsuhiko Ooyama ${ }^{2}$, Keiichi Kojima², Hisanori Kachi ${ }^{2}$ \\ Tatsuichirou $\mathrm{Abe}^{2}$, Kazutoshi Amano ${ }^{1}$ and Toshiaki Aoyama ${ }^{2}$ \\ ${ }^{1}$ Idemitsu Technofine Co.,Ltd. (SI BIdg. Aoyama, 1-3-6 Kita-Aoyama, Minato-Ku, Tokyo 107-0061, JAPAN) \\ ${ }^{2}$ Central Research Laboratory, The Nisshin OilliO Group, Ltd.(1 Shinmei-cho, Yokosuka, Kanagawa 239-0832, JAPAN)
}

\begin{abstract}
Disruption of the skin barrier function caused by epidermal hyper-proliferation, results in the skin becoming dry and showing high transepidermal water loss (TEWL). Gamma linolenic acid (GLA) is reportedly efficacious for treating TEWL and epidermal hyper-proliferation. In this study, to elucidate the effect of GLA-rich oil on skin function, GLA-containing food was given to adults with dry skin or mild atopic dermatitis and skin parameters were evaluated. In the results, we recognized beneficial effects on the TEWL index. The efficacy of GLA was also demonstrated to be statistically significant especially in subjects with pro-inflammatory features. The results suggest that the mechanism of improvement of skin barrier has been associated with possible generation of anti-inflammatory metabolites from GLA. The clinical physician also confirmed that none of the subjects showed any noteworthy side effects. GLA-enriched food appears to be safe and to improve skin barrier function in subjects with dry skin conditions and mild atopic dermatitis.
\end{abstract}

Key words: gamma-linolenic acid, dry skin, anti-inflammatory, human

\section{INTRODUCTION}

The mammalian epidermis is a stratified epithelium, one of the main functions of which is to generate a barrier, i.e. a relatively impermeable layer of dead cells, designed to protect the organism from dehydration and environmental stress. The epidermal barrier is composed of intercellular lipid lamellae with ceramides, cholesterol and fatty acids in the stratum corneum. Any barrier disruption increases transepidermal water loss (TEWL) and epidermal hyperproliferation, both of which are observed in abnormal skin conditions such as essential fatty acid deficiency ${ }^{1,2)}$.

Gamma linolenic acid (GLA: 18:3n-6), which is metabolized from linoleic acid (LA: 18:2n-6), is known for its beneficial effect such as improvement of lipid profiles ${ }^{3)}$ or alleviation of premenstrual syndrome (PMS) ${ }^{4}$. And GLA is also one of the most popular treatments available for dermatitis. Chung et al. investigated reversal of epidermal hyper-proliferation by GLA supplementation ${ }^{5)}$. Brosche et al. also demonstrated that, in elderly people, consuming GLA-rich borage oil altered fatty acid metabolism and improved TEWL $^{6)}$. These and other reports strongly suggest that GLA supplementation may contribute to improving epider- mal barrier function by lowering TEWL and preventing hyper-proliferation.

Prostaglandin $\mathrm{E}_{1}\left(\mathrm{PGE}_{1}\right)$ and 15-hydroxyeicosatrienoic acid(15-HETrE) converted from GLA via di-homo-gamma linolenic acid (DGLA: 20:3n-6) has anti-inflammatory properties. On the other hand, arachidonic acid (AA: 20:4n-6) is a precursor of prostaglandins $(\mathrm{PG})$ of the 2 -series such as $\mathrm{PGE}_{2}$ and leukotriene (LT) $\mathrm{B}_{4}$ which exert hyper-proliferative and inflammatory actions ${ }^{7}$. 15 -HETrE is a potent inhibitor of $\mathrm{LTB}_{4}$ generation and improves skin barrier function $^{8-10)}$.

One of the anti-inflammatory factors, the alimentary availability of GLA, is in fact limited to human milk and certain herbal seeds. GLA is otherwise unavailable in ordinary foods. The oral supplementation of cretin seed oil rich in GLA, particularly evening primrose oil, results in favorable improvement in serum profiles of the intermediates of GLA. On the other hand, the first hepatic step in the metabolism of LA, 6-desaturation to GLA, has been shown to be highly vulnerable to a variety of factors that may affect the condition of the $\operatorname{skin}^{11}$.

During the search for a way to industrially produce

\footnotetext{
*Correspondence to: Asuka Kawamura, Technology Development Dept., Idemitsu Technofine Co., Ltd. SI Bldg. Aoyama, 1-3-6 Kitaaoyama, Minato-Ku, Tokyo, 107-0061, JAPAN

E-mail: asuka.kawamura@itf.idemitsu.co.jp

Accepted October 25, 2011 (received for review August 19, 2011)

Journal of Oleo Science ISSN 1345-8957 print / ISSN 1347-3352 online

http://www.jstage.jst.go.jp/browse/jos/ http://mc.manusriptcentral.com/jjocs
} 
physiologically important fatty acids by the fermentation processes, Amano et al. found that fungi, Mucor circinelloides, accumulated GLA in a medium of up to $20 \%$ triacylglycerol ${ }^{12)}$. To elucidate the effect of this GLA-rich oil on skin function, this mold oil was incorporated into foods and administered to 130 adults. In contrast to evening primrose oil, the mold oil is less desaturated and has good oxidation stability. In the present study, this mold oil in food was considered in connection with aromas such as that of eicosapentaenoic acid (EPA), a well known anti-inflammatory precursor.

To investigate effects of GLA supplementation, we administered GLA-containing foods to subjects with dry skin and mild atopic dermatitis (AD) for 12 weeks. Skin parameters, especially TEWL and water content of the epidermis were then measured. Furthermore, to confirm the relationship between GLA and inflammation, we focused on subject groups based on levels of inflammatory compounds, i.e. low GLA or DGLA (precursor of anti-inflammatory eicosanoids) and high AA (precursor of pro-inflammatory eicosanoids).

\section{EXPERIMENTAL PROCEDURES}

\subsection{Subjects}

The protocol of this study was approved by the Ethics Review Board, Kensyoukai Medical Corporation(Osaka, Japan), and the study was performed in accordance with the Declaration of Helsinki. The subjects were adult males and females leading healthy social lives and who responded to a recruiting advertisement for paid subjects of clinical trials by DRC Co. Ltd. (Osaka, Japan) and TTC Co. Ltd. (Tokyo, Japan). We selected 130 subjects with dry skin (corneometer value $<50$ a.u. ${ }^{14)}$ ) and mild to moderate $\mathrm{AD}$ diagnosed according to the criteria of the guidelines for the treatment of $\mathrm{AD} 2009$ by committee for guidelines for management of atopic dermatitis of Japanese dermatological association. Subjects with the following conditions were excluded: AD patients taking steroids, using a specific health food regularly other than the test diets, showing an allergic reaction to the test diets, being continuously treated by a physician, having severe renal dysfunction, severe liver dysfunction, severe anemia, endocrine disorders, planning pregnancy and nursing during the study period, planning to undergo anesthesia during the study period, or having any concern about developing pollinosis during the study period. Any subject judged ineligible by a physician was also excluded. The subjects recorded all foods consumed during the study period. Subjects were instructed not to diet excessively or to overeat, and excessive sunbathing was also discouraged. Six subjects with a personal reason and 2 who took other therapeutic medications (anti-allergic drugs) discontinued the study at the discretion of the physician. Thus, data from 112 subjects were analyzed in this study.

\subsection{Test foods}

Test foods were cream sandwich wafers containing the test oil or control oil. Table 1 shows the nutritional compositions of the test foods. The GLA-enriched oil (test oil)was extracted from the fungus Mucor circinelloides. Common blended oil, a mixture of rapeseed and soybean oils, was used as a control. The fatty acid compositions of the test and control oils were determined employing a gas chromatography system (6890 series, Agilent Technologies, CA, USA) with a capillary column (TC-70, GL-Science, Tokyo, Japan), after methylation with boron trifluoride ${ }^{13)}$. The assay of the GLA content as a free fatty acid was performed using heptadecaoic acid as the internal standard. Table 2 shows the fatty acid compositions of the test and control oils. The test and control cream sandwich wafers were identical in appearance and taste.

\subsection{Study design}

A double-blind controlled study was performed. Before the study, the subjects were divided into two groups by an individual not directly involved in the study. The two groups were similar with respect to corneometer values and gender distributions. Another individual without

Table 1 Participant and nutritional compositions of test foods (daily intake: 2 sheets).

\begin{tabular}{cccc}
\hline \multicolumn{1}{c}{ Component } & & Control Diet & Test Diet \\
\hline Participant composition & GLA & $0.06 \mathrm{mg}$ & $200 \mathrm{mg}$ \\
& & & \\
Nutritional composition & Calorie & $104.0 \mathrm{Kcal}$ & $104.4 \mathrm{Kcal}$ \\
& Protein & $0.72 \mathrm{~g}$ & $0.72 \mathrm{~g}$ \\
& Lipid & $5.94 \mathrm{~g}$ & $6.02 \mathrm{~g}$ \\
& Carbohydrate & $11.92 \mathrm{~g}$ & $11.86 \mathrm{~g}$ \\
& Sodium & $15.62 \mathrm{mg}$ & $15.48 \mathrm{mg}$ \\
\hline
\end{tabular}


Table 2 Fatty acid composition and $\gamma$-linoleic acid content of the control and test oils.

\begin{tabular}{lcr}
\hline & Control oil & Test oil \\
\hline Fatty acid composition & (g/100g total fatty acids $)$ \\
\hline $14: 0^{1}$ & 0.1 & 0.9 \\
$16: 0$ & 7.5 & 10.9 \\
$16: 1$ & 0.2 & 1.6 \\
$18: 0$ & 3.0 & 1.4 \\
$18: 1$ & 42.5 & 38.1 \\
$18: 2$ & 36.0 & 9.9 \\
$18: 3 \alpha$ & 7.3 & 0.1 \\
$18: 3 \gamma$ & 0.0 & 28.7 \\
$20: 0$ & 0.5 & 0.1 \\
$20: 1$ & 0.6 & 0.8 \\
$22: 0$ & 0.4 & 0.1 \\
$22: 1$ & 0.1 & 0.1 \\
$24: 0$ & 0.2 & 0.7 \\
$24: 1$ & 0.1 & 0.3 \\
Others & 1.5 & 6.3 \\
Total & 100.0 & 100.0 \\
\hline$\gamma$-Linoleic acid content & $(\mathrm{g} / 100 \mathrm{~g}$ oil $)$ & \\
\hline $18: 3 \gamma$ & 0.0 & 25.0 \\
\hline
\end{tabular}

${ }^{1}$ Number of carbon atoms: number of double bonds.

access to the data for the groups allocated them to receive either the placebo or the test substance. A 4-week observation period before initiation of the test diet ingestion and a 4-week follow-up period after the test period were established. To observe influences of dietary nutrients, food records were kept with detailed descriptions for every food consumed during the study period. The subject recorded daily physical conditions and any special activities (e.g. intense exercise or sunburn) in a diary.

Skin hydration and TEWL measurements, blood tests, urinalysis, survey of subjective symptoms and examination by a physician were performed. The subjects were fasted 6 hours before tests. Then, skin hydration and TEWL were measured, and blood and urine were collected in the fasting state on the test day.

\subsection{Measurements}

Measurements were taken before treatment ( 0 week) and at 4, 8, 12 and 16 weeks after treatment.

2.4.1 The skin measurement

Skin hydration (arbitrary units) was determined by corneometry (Corneometer CM 825; Courage \& Khazaka Electronics, Cologne, Germany); TEWL (g/h per $\mathrm{m}^{2}$ ) was measured using a TEWA-Meter TM 300 (Courage \& Khazaka
Electronics). Subject skin conditions were diagnosed (desquamation, crusting) by a dermatologist. In addition, subjects recorded subjective symptoms of nocturnal and diurnal itching. Blood tests and urinalysis were entrusted to a commercial laboratory (Kishimoto Clinical Laboratory Group, Tomakomai, Japan). Before the measurements were obtained, the subjects were allowed to adapt for 20 min to a room temperature of $21 \pm 1^{\circ} \mathrm{C}$ and a relative humidity of $50 \pm 5 \%$.

2.4.2 Plasma fatty acid composition analysis and stratified analysis of skin evaluation results by level

We performed fatty acid composition analyses using subject plasma samples $(0 \mathrm{~W}, 12 \mathrm{~W})$. The measurements were carried out according to a fixed rule by SRL, Inc (Tokyo, Japan). In addition, for each subject, we calculated the contents of fatty acids (GLA, DGLA, AA) contributing to inflammation based on plasma analysis data from $0 \mathrm{~W}$ samples. Furthermore, for each fatty acid, we stratified based on the median for the entire subject population such that the subjects were almost equally divided. Furthermore, we divided the entire subject population into two groups, a pro-inflammatory group (GLA $<0.3 \%$, DGLA $\leqq$ $1.2 \%, \mathrm{AA}>6.2 \%)$ and an anti-inflammatory group $(0.3 \% \leqq$ GLA, DGLA $>1.2 \%, \mathrm{AA} \leqq 6.2 \%)$. For each stratification group, we performed a stratified analysis based on skin measurement data (TEWL and corneometer data).

\subsubsection{Subjective symptoms of itching; observation of skin} symptoms

As to the itching symptom, we evaluated intensity, range and frequency. We evaluated the intensity of the itching (nocturnal) using the visual analogue scale (VAS) score. "No sleep disorder" was given a score of 0 and "I am itchy and cannot sleep at all" was given a score of 100. Based on the evaluation of nocturnal itch using this score, "I am itchy, and it is almost intolerable" was given a score of 4, "I am itchy and wake up" was given a score of 3, "I can sleep if I scratch" was given a score of 2, "I can sleep even if I do not scratch" was given a score of 1 , and "I feel hardly any itching" was given a score of 0 . As to the frequency of itching, "More than two days/week" was given a score of 2, "One day/week" a score of 1 and "There was no itching" a score of 0 .

In the observation of skin manifestations, we rated the physician's judgment of atopic state on a five point scale; "no symptoms" was given a score of 0 , "mild symptoms" was given a score of 1 , "moderate symptoms" was given a score of 2, "severe symptoms" was given a score of 3 and "more severe symptoms" was given a score of 4 . Desiccation, desquamation and curettage were assessed by applying a four point scale; " - " is a score of 0, " + " is a score of 1 , " ++ " is a score of 2 and " +++ " is a score of 3. Elements of papule formation were assessed on a four point scale; "no symptoms" is a score of 0 , "mild symptoms" is a score of 1 , "moderate symptoms" is a score of 2 , and 
"severe symptoms" is a score of 3. Areas of eruption were evaluated on a three point scale; "none" is a score of 0 , "less than one-third of the body surface" is a score of 1 , "one-third to two-thirds of the body surface" is a score of 2 and "more than two-thirds" is a score of 3 .

2.4.4 Blood test and urinalysis

Hematological tests included white blood cells (WBC), red blood cells $(\mathrm{RBC})$, hemoglobin $(\mathrm{Hb})$, hematocrit $(\mathrm{Ht})$ and platelets (PLT). Biochemical tests were conducted to measure aspartate transaminase (AST; GOT: glutamic oxalic transaminase), alanine transaminase (ALT; GPT: glutamic-pyruvate transaminase), lactate dehydrogenase (LDH), alkaline phosphatase (ALP), gamma-glutamyltranserase (g-GTP), total bilirubin (T-Bil), albumin (Alb), total protein (TP), blood urea nitrogen (BUN), creatinine (Cre), uric acid (UA), total cholesterol (TC), high-density lipoprotein cholesterol (HDL-C), low-density lipoprotein cholesterol LDL-C, triglycerides (TG), glucose (Glu), glycosylated hemoglobin $(\mathrm{HbA1C})$, sodium $(\mathrm{Na})$, potassium $(\mathrm{K})$ and chloride $(\mathrm{Cl})$. Urinalysis was performed to determine specific gravity, $\mathrm{pH}$, sugar, occult blood, protein, urobilinogen and ketone bodies. All of these measurements were performed by a commercial laboratory (Kishimoto Clinical Laboratory Group, Tomakomai, Japan).

\subsection{Statistical analysis}

The measured values are presented as means \pm standard deviation excluding values in the figures. The measured values in the figures are presented as means \pm standard error. For comparison of TEWL, stratum corneum hydration and the VAS of itching, data obtained from the two groups before and after the test diet ingestion were employed. The unpaired or paired $t$-test was used for statistical comparisons. As to pruritus intensity and the frequency of subjective symptoms of itching and skin condition judgments made by physicians before and after the test diet ingestion, the Mann-Whitney U test or Wilcoxon signed-ranks test was used for comparison.

\section{RESULTS}

\subsection{Characteristics of subjects}

Characteristics of subjects in the efficacy and safety trials are shown in Tables 3 and 4, respectively. All mean values of height, body weight, systolic blood pressure, diastolic blood pressure and heart rate before test diet ingestion remained within normal and standard ranges. Some clinical test values were outside of reference ranges in a portion of the subjects, but they were still selected for the study after the physician judged them to be appropriate for

Table 3 Characteristics of subjects (efficacy trial) ${ }^{1}$.

\begin{tabular}{|c|c|c|c|c|}
\hline & Group & All participants & Healthy Subjects & Patients with $\mathrm{AD}$ \\
\hline & $\mathrm{n}$ & 122 & 66 & 56 \\
\hline & & 61 (GLA) & 32 (GLA) & 29 (GLA) \\
\hline & & 61 (Control) & 34 (Control) & 27 (Control) \\
\hline \multirow[t]{2}{*}{ Age (years) } & GLA & $36.7 \pm 11.6$ & $41.0 \pm 11.8$ & $31.9 \pm 9.4$ \\
\hline & Control & $36.5 \pm 11.6$ & $41.0 \pm 11.8$ & $30.8 \pm 8.6$ \\
\hline \multirow{2}{*}{ Height $(\mathrm{cm})$} & GLA & $161.02 \pm 8.22$ & $159.48 \pm 7.63$ & $162.72 \pm 8.64$ \\
\hline & Control & $160.94 \pm 8.32$ & $159.79 \pm 7.99$ & $162.37 \pm 8.66$ \\
\hline \multirow[t]{2}{*}{ Body weight (kg) } & GLA & $58.19 \pm 12.73$ & $56.80 \pm 9.46$ & $59.72 \pm 15.61$ \\
\hline & Control & $55.72 \pm 12.20$ & $55.00 \pm 11.65$ & $56.62 \pm 13.03$ \\
\hline \multirow[t]{2}{*}{ Body mass index $\left(\mathrm{kg} / \mathrm{m}^{2}\right)$} & GLA & $22.34 \pm 3.88$ & $22.36 \pm 3.65$ & $22.32 \pm 4.17$ \\
\hline & Control & $21.34 \pm 3.16$ & $21.35 \pm 2.77$ & $21.34 \pm 3.64$ \\
\hline \multirow{2}{*}{$\begin{array}{l}\text { Systolic blood pressure } \\
(\mathrm{mmHg})\end{array}$} & GLA & $116.1 \pm 14.4^{\#}$ & $116.2 \pm 14.1$ & $116.0 \pm 14.9$ \\
\hline & Control & $111.3 \pm 12.2$ & $112.0 \pm 11.0$ & $110.3 \pm 13.8$ \\
\hline \multirow{2}{*}{$\begin{array}{l}\text { Diastolic blood pressure } \\
(\mathrm{mmHg})\end{array}$} & GLA & $70.0 \pm 10.0$ & $70.6 \pm 9.0$ & $69.3 \pm 11.2$ \\
\hline & Control & $67.0 \pm 8.1$ & $68.6 \pm 8.3$ & $65.0 \pm 7.5$ \\
\hline \multirow[t]{2}{*}{ Heart rate (bpm) } & GLA & $68.7 \pm 10.9$ & $67.2 \pm 10.7$ & $70.3 \pm 11.2$ \\
\hline & Control & $70.4 \pm 8.7$ & $69.9 \pm 8.7$ & $71.0 \pm 8.8$ \\
\hline
\end{tabular}

Daily GLA intake was $0.06 \mathrm{mg}$ in the control group and $200 \mathrm{mg}$ in the GLA group.

${ }^{1}$ Values are means \pm SD.

Significantly different from the control group value, at ${ }^{\#} P<0.05$ by unpaired $t$-test. 
Table 4 Characteristics of subjects (safety trial) ${ }^{1}$.

\begin{tabular}{|c|c|c|c|c|}
\hline & Group & All participants & Healthy Subjects & Patients with $\mathrm{AD}$ \\
\hline & $\mathrm{n}$ & 124 & 66 & 58 \\
\hline & & 61 (GLA) & 32 (GLA) & 29 (GLA) \\
\hline & & 63 (Control) & 34 (Control) & 29 (Control) \\
\hline \multirow[t]{2}{*}{ Age (years) } & GLA & $36.7 \pm 11.6$ & $41.0 \pm 11.8$ & $31.9 \pm 9.4$ \\
\hline & Control & $36.5 \pm 11.4$ & $41.0 \pm 11.8$ & $31.3 \pm 8.6$ \\
\hline \multirow[t]{2}{*}{ Height $(\mathrm{cm})$} & GLA & $161.02 \pm 8.22$ & $159.48 \pm 7.63$ & $162.72 \pm 8.64$ \\
\hline & Control & $160.88 \pm 8.20$ & $159.79 \pm 7.99$ & $162.16 \pm 8.40$ \\
\hline \multirow[t]{2}{*}{ Body weight (kg) } & GLA & $58.19 \pm 12.73$ & $56.80 \pm 9.46$ & $59.72 \pm 15.61$ \\
\hline & Control & $55.94 \pm 12.29$ & $55.00 \pm 11.65$ & $57.03 \pm 13.13$ \\
\hline \multirow[t]{2}{*}{ Body mass index $\left(\mathrm{kg} / \mathrm{m}^{2}\right)$} & GLA & $22.34 \pm 3.88$ & $22.36 \pm 3.65$ & $22.32 \pm 4.17$ \\
\hline & Control & $21.45 \pm 3.27$ & $21.35 \pm 2.77$ & $21.56 \pm 3.83$ \\
\hline \multirow{2}{*}{$\begin{array}{l}\text { Systolic blood pressure } \\
(\mathrm{mmHg})\end{array}$} & GLA & $116.1 \pm 14.4$ & $116.2 \pm 14.1$ & $116.0 \pm 14.9$ \\
\hline & Control & $111.4 \pm 12.5$ & $112.0 \pm 11.0$ & $110.7 \pm 14.2$ \\
\hline \multirow{2}{*}{$\begin{array}{l}\text { Diastolic blood pressure } \\
(\mathrm{mmHg})\end{array}$} & GLA & $70.0 \pm 10.0$ & $70.6 \pm 9.0$ & $69.3 \pm 11.2$ \\
\hline & Control & $67.3 \pm 8.4$ & $68.6 \pm 8.3$ & $65.9 \pm 8.4$ \\
\hline \multirow[t]{2}{*}{ Heart rate (bpm) } & GLA & $68.7 \pm 10.9$ & $67.2 \pm 10.7$ & $70.3 \pm 11.2$ \\
\hline & Control & $70.4 \pm 8.6$ & $69.9 \pm 8.7$ & $71.0 \pm 8.7$ \\
\hline
\end{tabular}

Daily GLA intake was $0.06 \mathrm{mg}$ in the control group and 200mg in the GLA group.

${ }^{1}$ Values are means \pm SD.

There were no significant differences between the groups.
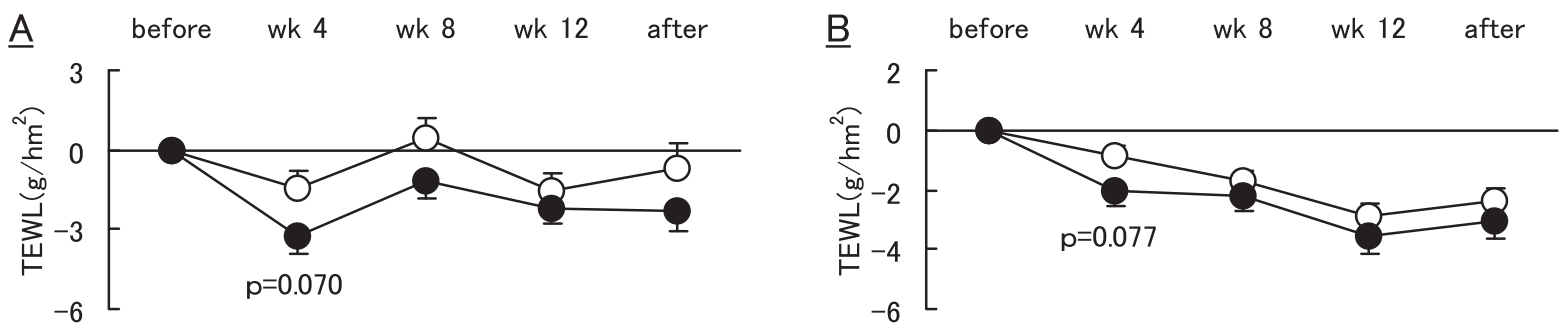

Fig. 1 Changes in transepidermal water loss at the cheek (A) and forearm (B) from baseline after intake of the control diet (open circles, $\mathrm{n}=61$ ) or GLA diet (closed circles, $\mathrm{n}=61$ ). The daily GLA intake was $0.06 \mathrm{mg}$ in the control group and $200 \mathrm{mg}$ in the GLA group. Each point represents the mean \pm standard error. $P$ values were obtained using the unpaired $t$-test for comparisons between groups.

participation in these trials.

\subsection{Efficacy trial}

\subsubsection{Transepidermal water loss}

Changes in TEWL are shown in Fig. 1. Although no significant differences were noted between the groups, TEWL of the cheek $(-1.47 \pm 5.25$ in the control group and -3.24 \pm 5.44 in the GLA group) and forearm $(-0.90 \pm 3.23$ in the control group and $-2.03 \pm 3.77$ in the GLA group) tended to be lower in the GLA than the control group at week 4 .

3.2.2 Stratum corneum hydration

Changes in stratum corneum hydration are shown in Fig. 2A (cheek) and 2B (forearm). No significant differences were noted between the two groups.

3.2.3 Compositions of plasma total fatty acids

Compositions of plasma GLA, DGLA and AA before the test diet ingestion and at week 12 are shown in Table 5. Plasma GLA was higher in the GLA than in the control group at week 12. Changes in plasma GLA and DGLA compositions in the GLA group were larger than those in the control group.

3.2.4 Transepidermal water loss (stratified data)

TEWL changes in groups of subjects stratified by plasma total fatty acid compositions before test diet ingestion are shown in Fig. 3 and 4(A: plasma GLA $<0.3 \%$, C: plasma DGLA $<1.2 \%$, E: plasma AA $>6.2 \%$; the stratified groups 

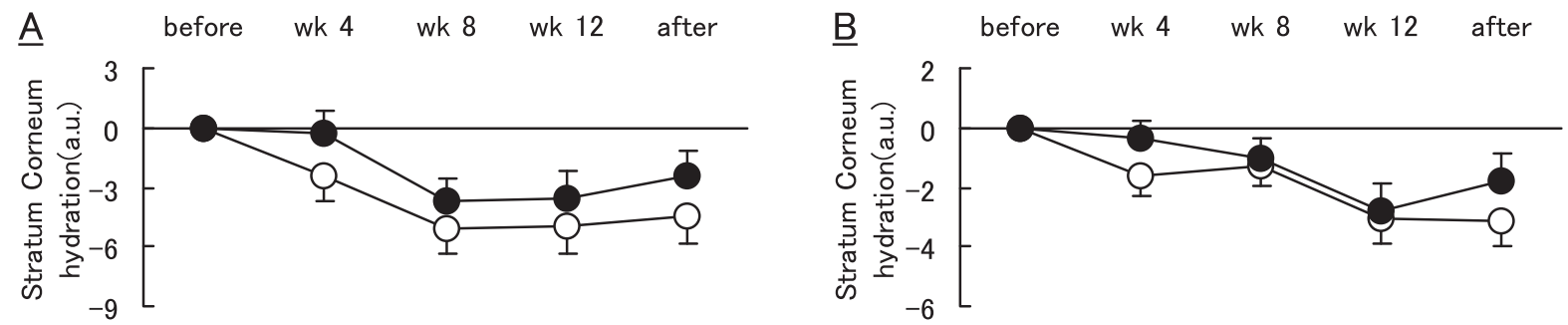

Fig. 2 Changes in stratum corneum hydration of the cheek (A) and forearm (B) from baseline after intake of the control diet (open circles, $n=61$ ) or GLA diet (closed circles, $n=61$ ). The daily GLA intake was $0.06 \mathrm{mg}$ in the control group and 200mg in the GLA group. Each point represents the mean \pm standard error. There were no significant differences between the groups.

Table 5 Plasma total fatty acid compositions ${ }^{1}$.

\begin{tabular}{lllll}
\hline & Group & \multicolumn{1}{c}{ Before } & \multicolumn{1}{c}{ Week 12 } & \multicolumn{1}{c}{$\Delta^{2}$} \\
\hline GLA (\%) & GLA & $0.35 \pm 0.17$ & $0.47 \pm 0.18^{\# * *}$ & $0.12 \pm 0.20^{\# \#}$ \\
& Control & $0.33 \pm 0.17$ & $0.34 \pm 0.15$ & $0.01 \pm 0.15$ \\
DGLA (\%) & GLA & $1.23 \pm 0.27$ & $1.42 \pm 0.28^{* *}$ & $0.19 \pm 0.30^{\#}$ \\
& Control & $1.25 \pm 0.37$ & $1.31 \pm 0.35$ & $0.06 \pm 0.31$ \\
AA (\%) & GLA & $6.23 \pm 1.08$ & $6.62 \pm 1.10^{* *}$ & $0.38 \pm 0.82$ \\
& Control & $6.22 \pm 1.22$ & $6.50 \pm 1.21^{*}$ & $0.28 \pm 0.84$ \\
\hline
\end{tabular}

The daily GLA intake was $0.06 \mathrm{mg}$ in the control group and $200 \mathrm{mg}$ in the GLA group.

${ }^{1}$ Values are means \pm SD (GLA: $n=61$, Control: $n=61$ ).

${ }^{2}$ Twelve-week value minus value of before the beginning of the study.

Significantly different compared with the value of the control group, at ${ }^{\#} P<0.05$ and

${ }^{\#} P<0.01$ by unpaired $t$-test.

Significantly different compared with the value before the beginning of the study, at

$* P<0.05$ and $* * P<0.01$ by paired $t$-test.

of subjects tended to be pro-inflammatory, B: plasma GLA $\geqq 0.3 \%$, D: plasma DGLA $>1.2 \%$, F: plasma $\mathrm{AA} \leqq 6.2 \%$; the stratified groups of subjects tended to be anti-inflammatory). As shown in Fig. 3C, in the stratified group of subjects whose plasma DGLA was $1.2 \%$ or less, the change in cheek TEWL of the GLA group $(-1.16 \pm 3.13)$ was significantly lower than in the control group $(1.41 \pm 5.80)$ at week 8. The change in forearm TEWL of the GLA group $(-2.37$ $\pm 2.70)$ was also lower than that in the control group ($0.50 \pm 3.50)$ at week 8 in the group of subjects stratified by plasma AA over $6.2 \%$ (Fig. 4E). These statistically significant differences attributable to the test diet were seen between pro-inflammatory groups. Conversely, no statistically significances or tendencies for improvement were observed between anti-inflammatory groups (Fig. 3 B, D, F, Fig. 4 B, D, F).

3.2.5 Stratum corneum hydration (stratified data)

There were no significant differences between the subject groups stratified by plasma total fatty acid compositions before the test diet ingestion (data not shown).

3.2.6 Subjective symptom of itching

There were no significant differences between the groups in the subjective symptom of diurnal itching ac- cording to the scores for VAS, pruritus intensity and frequency (data not shown).

Compared with the baseline, the VAS of nocturnal itching in the GLA group was reduced (i.e. improved) significantly at week 8 . The scores for pruritus intensity and frequency of nocturnal itching were also reduced at weeks 4 and 8 in the GLA group. However, no such statistically significant changes were seen in the control group during the same periods (Table 6). For all items pertaining to these subjective symptoms, there were no time points at which significant improvements were observed in the control group as compared with the GLA group.

3.2.7 Judgment of skin manifestations (data not shown)

There were no significant differences between groups in the judgment of skin manifestations (desiccation, desquamation, curettage, physician's judgment of atopic state, physician's judgment of itemized atopic state; erythema and acute phase papules, eczema and crusting, chronic phase papules, nodules, lichenification, area of eruption, and the sum of these items). 

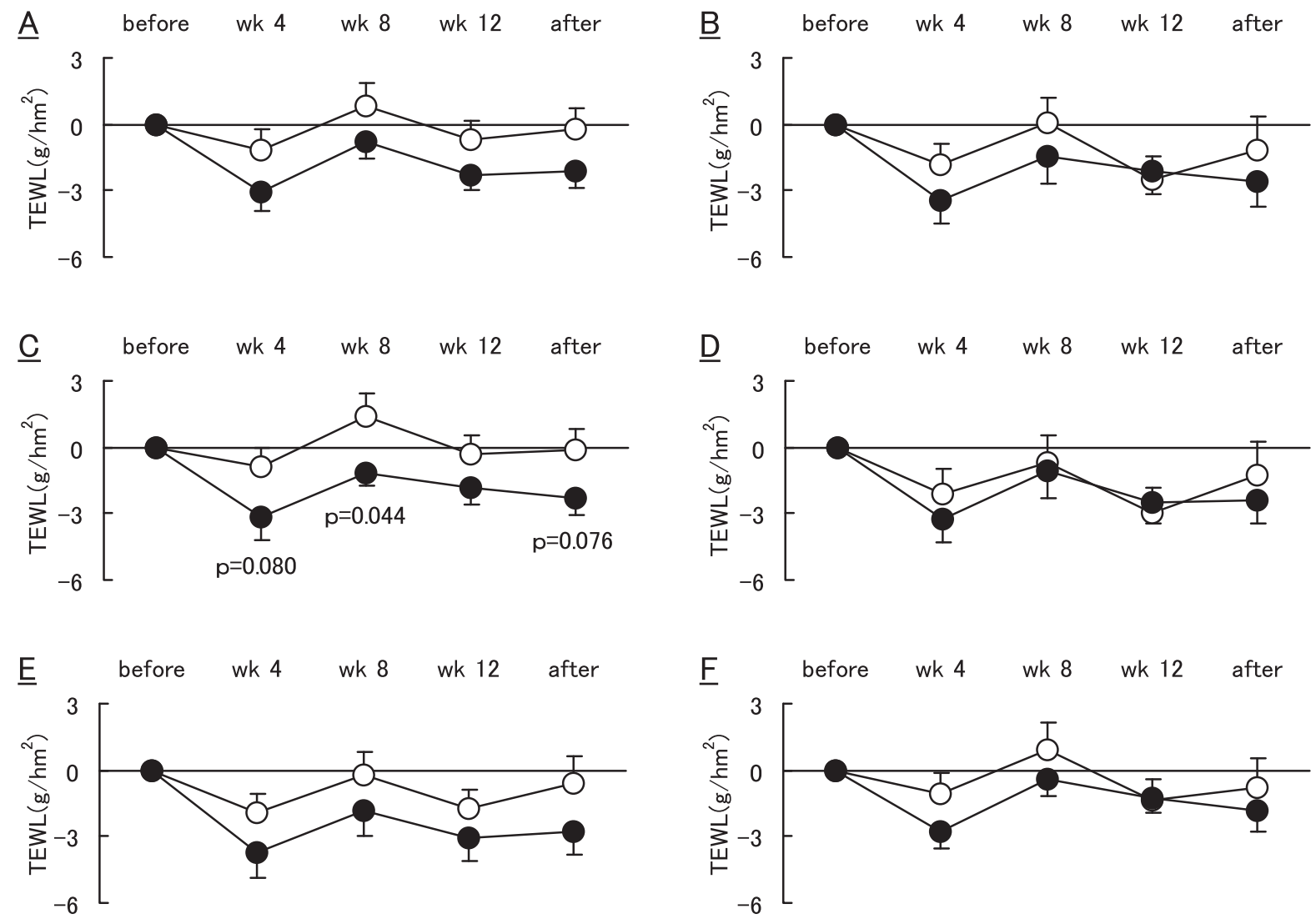

Fig. 3 Changes in transepidermal water loss at the cheek from baseline after intake of the control diet (open circles) or GLA diet (closed circles) in subjects whose composition of plasma GLA was less than $0.3 \%$ (A, GLA: $n=31$, Control: $n=32$ ) or above $0.3 \%$ (B, GLA: $n=30$, Control: $n=29$ ), plasma DGLA was $1.2 \%$ or less (C, GLA: $n=27$, Control: $\mathrm{n}=32$ ) or over $1.2 \%$ (D, GLA: $\mathrm{n}=34$, Control: $\mathrm{n}=29$ ), and plasma AA was over $6.2 \%$ (E, GLA: $\mathrm{n}=31$, Control: $n=27$ ) or $6.2 \%$ or less (F, GLA: $n=30$, Control: $n=34$ ). Participants stratified by A, C and E were designated as "pro-inflammatory", B, D and F as "anti-inflammatory". The daily GLA intake was $0.06 \mathrm{mg}$ in the control group and $200 \mathrm{mg}$ in the GLA group. Each point represents the mean \pm standard error. $P$ values were obtained using the unpaired $t$-test for comparisons between groups.

\subsection{Safety trial}

3.3.1 Physical measurement (data not shown)

Significant changes were observed in physical measurement items excluding mean systolic blood pressure during the 12-week test period and the 4-week follow-up period as compared with the values of before test diet ingestion. However, all of these changes were slight and the physician judged all of them as having no clinical importance.

\subsubsection{Adverse events (data not shown)}

No critical or significant episodes were observed and no adverse events attributable to the test diet were noted by the monitoring physician. Although some clinical test values were outside of reference ranges in a portion of the subjects, these were temporary and minor. Thus, the physician judged these changes to be minor events not related to the test substance.

3.3.3 Blood test and urinalysis (data not shown)

Although significant changes were observed on hemato- logical tests, blood biochemical tests and urinalysis after as compared with before test diet ingestion, all of the values remained within reference ranges. Hence, the monitoring physician confirmed that there were no clinical problems related to any of these laboratory value changes.

\subsection{Usage of medical agents (data not shown)}

Eight subjects ( 2 healthy subjects and 6 patients with $\mathrm{AD})$ in the GLA group and 5(1 healthy subject and 4 patients with $\mathrm{AD}$ ) in the control group used steroids, tacrolimus or moisturizers during the test period. Steroid dosages during the test period were not changed significantly as compared with before the test diet ingestion. One subject each used tacrolimus or moisturizer and there were no major changes in the dosages of these agents. One subject used a steroid preparation as well as tacrolimus. 

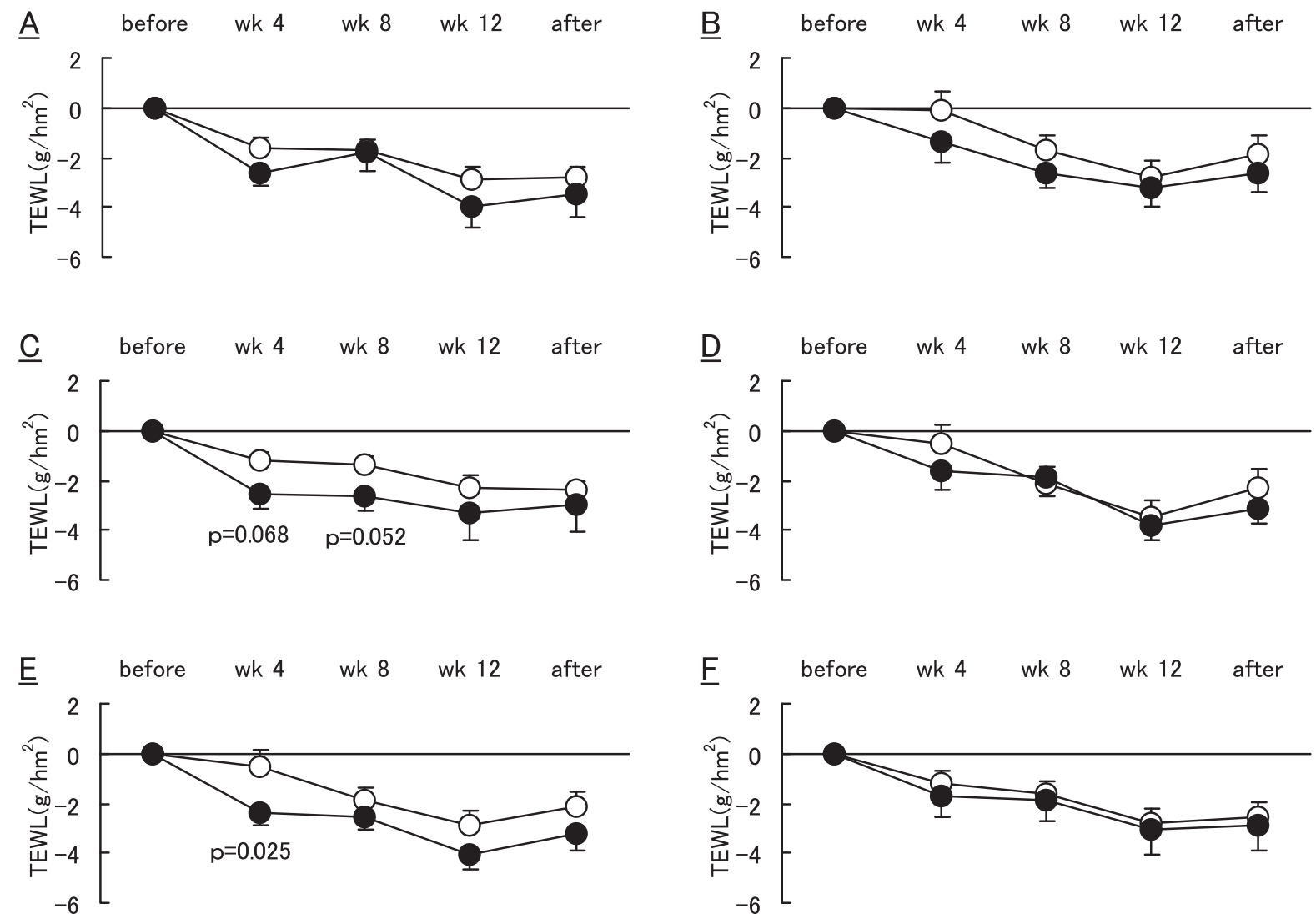

Fig. 4 Changes in transepidermal water loss at the forearm from baseline after intake of the control diet (open circles) or GLA diet (closed circles) in subjects whose composition of plasma GLA was less than $0.3 \%$ (A, GLA: $n=31$, Control: $n=32$ ) or above $0.3 \%$ (B, GLA: $n=30$, Control: $n=29$ ), plasma DGLA was $1.2 \%$ or less (C, GLA: $n=27$, Control: $\mathrm{n}=32$ ) or over $1.2 \%$ (D, GLA: $\mathrm{n}=34$, Control: $\mathrm{n}=29$ ), and plasma AA was over $6.2 \%$ (E, GLA: $\mathrm{n}=31$, Control: $n=27$ ) or $6.2 \%$ or less (F, GLA: $n=30$, Control: $n=34$ ). Participants stratified by A, C and E were designated as "pro-inflammatory", B, D and F as anti-inflammatory. The daily GLA intake was $0.06 \mathrm{mg}$ in the control group and $200 \mathrm{mg}$ in the GLA group. Each point represents the mean \pm standard error. $P$ values were obtained using the unpaired $t$-test for comparisons between groups.

\section{DISCUSSION}

TEWL is known to be high in skin rashes such as AD or psoriasis $^{15,16)}$. Morse et al. analyzed 26 clinical studies including 1207 atopic patients and established that GLA-rich oil has a beneficial effect on atopic eczema ${ }^{17)}$. Kragballe $e t$ $a l$. showed that dietary supplementation with a combination of n-3 fatty acids and GLA was useful for the treatment of psoriasis ${ }^{18)}$. Yen et al. reported that $\mathrm{AD}$ patients tended to have lower levels of GLA and DGLA than controls and that TEWL correlated negatively with serum levels of LA metabolites such as GLA and DGLA. Consequently, it was concluded that deficiencies in n-6 essential fatty acids correlated with the severity of skin barrier dysfunction and cutaneous inflammation ${ }^{19)}$.

In this study, to investigate effect of GLA supplementation, GLA-containing foods were administered to subjects with dry skin and mild $\mathrm{AD}$ and skin parameters, especially TEWL and water content of the epidermis were measured.
After 4 weeks, the primary efficacy analyses of skin parameters demonstrated a beneficial effect in the GLA diet group as compared with the control diet group (Fig. 1, cheek: $p=0.070$, forearm: $p=0.077)$. Although there were no significant differences in other time or stratum corneum hydration indices throughout the test period, the GLA diet group showed satisfactory skin parameters (low TEWL and high stratum corneum index) as compared with the control diet group. Thus, our preliminary results suggest that GLA might improve skin properties and protect the skin barrier.

GLA efficiently reverses epidermal hyper-proliferation due to GLA metabolites such as anti-inflammatory eicosanoids which exert anti-proliferative properties and these actions are associated with an increase in ceramide synthesis and improvement of skin barrier function ${ }^{5)}$. Thus, patients with high levels of pro-inflammatory eicosanoids and/or low levels of anti-inflammatory eicosanoids appear to be more responsive to the beneficial effects of GLA sup- 
Table 6 Subjective symptom of nocturnal itching ${ }^{1}$.

\begin{tabular}{|c|c|c|c|c|c|c|c|}
\hline & & Group & Before & Week 4 & Week 8 & Week 12 & After \\
\hline \multirow[t]{4}{*}{ VAS } & & GLA & $22.6 \pm 26.5$ & $16.0 \pm 23.9^{* *}$ & $14.5 \pm 21.9^{* *}$ & $15.6 \pm 22.8^{*}$ & $16.2 \pm 24.9^{*}$ \\
\hline & & Control & $22.1 \pm 25.3$ & $16.3 \pm 21.5^{* *}$ & $17.8 \pm 23.3$ & $16.3 \pm 22.7^{*}$ & $16.4 \pm 22.5^{*}$ \\
\hline & $\Delta^{2}$ & GLA & & $-6.6 \pm 16.2^{* *}$ & $-8.1 \pm 18.2^{* *}$ & $-7.0 \pm 21.0^{*}$ & $-6.4 \pm 23.9^{*}$ \\
\hline & & Control & & $-5.8 \pm 15.4^{* *}$ & $-4.2 \pm 22.1$ & $-5.8 \pm 18.5^{*}$ & $-5.7 \pm 20.7^{*}$ \\
\hline Pruritus & & GLA & $1.1 \pm 0.9$ & $0.9 \pm 0.9^{\text {क }}$ & $0.8 \pm 0.9^{\ddagger}$ & $0.8 \pm 0.9^{\dagger}$ & $0.9 \pm 1.0$ \\
\hline intensity & & Control & $1.1 \pm 0.9$ & $1.0 \pm 0.9$ & $0.9 \pm 0.9$ & $0.9 \pm 1.0^{\dagger}$ & $0.8 \pm 0.9^{\dagger}$ \\
\hline \multirow[t]{2}{*}{ (Score) } & $\Delta$ & GLA & & $-0.2 \pm 0.6^{\text {\# }}$ & $-0.3 \pm 0.7^{\ddagger}$ & $-0.3 \pm 0.9^{\dagger}$ & $-0.2 \pm 0.9$ \\
\hline & & Control & & $-0.1 \pm 0.7$ & $-0.2 \pm 0.9$ & $-0.2 \pm 0.8^{\dagger}$ & $-0.3 \pm 0.9^{\dagger}$ \\
\hline \multirow[t]{4}{*}{ Frequency } & & GLA & $1.2 \pm 0.9$ & $0.9 \pm 0.9^{\dagger}$ & $0.8 \pm 0.9^{\text {专 }}$ & $0.9 \pm 0.9$ & $0.9 \pm 0.9^{\dagger}$ \\
\hline & & Control & $1.1 \pm 0.9$ & $1.0 \pm 0.9$ & $1.0 \pm 0.9$ & $1.0 \pm 0.9$ & $1.0 \pm 0.9$ \\
\hline & $\Delta$ & GLA & & $-0.2 \pm 0.7^{\dagger}$ & $-0.3 \pm 0.9^{\text {क }}$ & $-0.2 \pm 1.0$ & $-0.2 \pm 0.8^{\dagger}$ \\
\hline & & Control & & $-0.1 \pm 0.7$ & $-0.2 \pm 0.9$ & $-0.2 \pm 0.9$ & $-0.2 \pm 0.8$ \\
\hline
\end{tabular}

The daily GLA intake was $0.06 \mathrm{mg}$ in the control group and 200mg in the GLA group.

${ }^{1}$ Values are means \pm SD (GLA: $\mathrm{n}=61$, Control: $\mathrm{n}=61$ ).

${ }^{2}$ Values at weeks $4,8,12$ and after the study minus the value before the beginning of the study.

Significantly different compared with the value before the beginning of the study, at $* P<0.05$ and $* * P<0.01$ by paired t-test.

Significantly different compared with the value before the beginning of the study, at ${ }^{\dagger} P<0.05$ and ${ }^{\ddagger} P<0.01$ by Wilcoxon signedranks test.

plementation.

To confirm the relationship between GLA and inflammatory processes, we focused on a group of subjects with proinflammatory features such as low levels of GLA or DGLA (precursor of anti-inflammatory eicosanoids) and high levels of AA (precursor of pro-inflammatory eicosanoids). As to TEWL in the pro-inflammatory group, two significant differences were seen in the GLA diet group as shown in Fig. $3 \mathrm{C}$ and $4 \mathrm{E}$, as compared with the control group. In contrast, the anti-inflammatory group showed no significant or beneficial effects (Fig. 3B, D, F, 4B, D, F). These results demonstrate the efficacy of GLA to be very strong in subjects with high levels of pro-inflammatory substances. Moreover, a strong relationship between the anti-inflammatory properties of GLA and improved skin barrier function was demonstrated. Like many other reports, this study also found that GLA supplementation did not increase AA $(\text { Table 5) })^{2,9,10,20,21)}$.

Additionally, the Wilcoxon signed-rank test showed improvement of the pruritus score among subjective symptoms, but there was no difference between the GLA diet group and the control group (Table 6). The clinical scores showed positive and negative tendencies and were not consistent (data not shown). Morse et al. reported that the effects on itching were particularly striking with GLA administration and the present results tended to be similar to theirs ${ }^{22}$. Most subjects in this study had dry skin but the atopic subjects had mild to moderate symptoms. These subjects were considered to have scores too low to show marked improvement. This might be why there was no pronounced propensity in the clinical findings and except for the pruritus index. Although how dietary GLA effects itching is not known, pruritus markedly affects quality of life and regulation of the itch control mechanism requires further investigation.

We found no significant differences at the level of the entire study population, possibly because some subjects with dry skin had conditions unrelated to fatty acid deficiency or inflammation. For example, increased TEWL was observed in non-filaggrin $\mathrm{AD}$ or sphingomyelinase impaired subjects $^{23,24)}$. In such subjects lacking the ability to metabolize ceramides and/or filaggrin due to genetic factors, impaired skin barrier function would not improve with only GLA supplementation and no protection from inflammatory processes would be provided by such dietary interventions.

In this study, we focused on skin barrier function and attempted to improve the skin barrier by administration of GLA. The results obtained within 4 weeks, as demonstrated by the primary efficacy analyses of skin parameters, confirmed a beneficial effect in the GLA diet group as compared with the control diet group. Therefore, significant differences were observed only in the group with high levels of pro-inflammatory compounds and in these subjects skin barrier function was demonstrated to be im- 


\section{A. Kawamura, K. Ooyama, K. Kojima et al.}

proved.

There were no concerns regarding the safety of the cream sandwich wafers containing GLA. Although we observed very slight differences, the monitoring physician confirmed that all items were inconsequential.

\section{CONCLUSION}

In conclusion, supplementation with GLA derived from fermentation was found to induce beneficial effects, i.e. improvement of skin barrier function, as reflected by reduced TEWL. Furthermore, statistically significant efficacy was demonstrated especially in subjects with high levels of pro-inflammatory substances. Therefore, anti-inflammatory eicosanoids delivered via GLA can enhance skin barrier function. GLA-enriched foods might safely play an important role in the skin barrier control.

\section{ACKNOWLEDGMENTS}

This study was supported by TTC Co., Ltd. (1-20-2 Ebisunishi, Shibuya-ku, Tokyo JAPAN) and the authors are grateful to Osamu Igarashi Ph.D. (Professor Emeritus of Ochanomizu University) for his valuable suggestions.

\section{References}

1) Kim, J.; Kim, H.; Jeong, do. H.; Kim, S. H.; Park, S. K.; Cho, Y. Comparative effect of gromwell (Lithospermum erythrorhizon) extract and borage oil on reversing epidermal hyperproliferation in guinea pigs. Biosci. Biotechnol. Biochem. 70, 2086-2095 (2006).

2) Ziboh, V. A.; Miller, C. C.; Cho, Y. Metabolism of polyunsaturated fatty acids by skin epidermal enzymes: generation of antiinflammatory and antiproliferative metabolites. Am. J. Clin. Nutr. 71, 361S-366S (2000).

3) Dasgupta S.; Bhattacharyya D. K. Dietary effect of $\gamma$-linolenic acid on the lipid profile of rat fed eruic acid rich oil. J. Oleo Sci. 56, 569-577(2007).

4) Watanabe S.; Sakurada M.; Tsuji H.; Matsumoto S.; Kondo K. Efficacy pf $\gamma$-linolenic acid for treatment of premenstrual syndrome, as assessed by a prospective daily rating system. J. Oleo Sci. 54, 214-224(2005).

5) Chung, S.; Kong, S.; Seong, K.; Cho, Y. Gamma-linolenic acid in borage oil reverses epidermal hyperproliferation in guinea pigs. J. Nutr. 132, 3090-3097 (2002).

6) Brosche, T.; Platt, D. Effect of borage oil consumption on fatty acid metabolism, transepidermal water loss and skin parameters in elderly people. Arch. Gerontol. Geriatr. 30, 139-150 (2000).

7) Bauer, F. W.; van de Kerkhof, P. C.; Maassen-de Grood,
R. M. Epidermal hyperproliferation following the induction of microabscesses by leukotriene B4. Br. J. Dermatol. 114, 409-412(1986).

8) Ziboh, V. A.; Naguwa, S.; Vang, K.; Wineinger, J.; Morrissey, B. M.; Watnik, M.; Gershwin, M. E. Suppression of leukotriene B4 generation by ex-vivo neutrophils isolated from asthma patients on dietary supplementation with gammalinolenic acid-containing borage oil: possible implication in asthma. Clin. Dev. Immunol. 11, 13-21 (2004).

9) Chilton-Lopez, Surette M. E.; Swan, D. D.; Fonteh, A.; Johnson, M. M.; Chilton, F. H. Metabolism of gammalinolenic acid in human neutrophils. J. Immunol. 156, 2941-2947(1996).

10) Iversen, L.; Fogh, K.; Bojese, G.; Kragballe, K. Linoleic acid and dihomogammalinolenic acid inhibit leukotriene B4 formation and stimulate the formation of their 15-lipoxygenase products by human neutrophils in vitro. Evidence of formation of antiinflammatory compounds. Agents Actions 33, 286-291 (1991).

11) Brenne, R. R. Nutritional and hormonal factors influencing desaturation of essential fatty acids. Prog. Lipid Res. 20, 41-47 (1981).

12) Kikutsugi, H.; Amano, K. The production and application of gamma-linolenic acid produced by microorganisms. Tech. J. Food Chem. \& Chem. 12, 47-52(1990).

13) Shirasawa, S.; Sasaki, A.; Saida, Y.; Satoh, C. A rapid method for trns-fatty acid determination using a single capillary GC. J. Oleo Sci. 56, 53-58(2007).

14) Heinrich U.; Koop U.; Leneveu-Duchemin M. C. Multicenter comparison of skin hydration in terms of physical-, physiological- and product-dependent parameters by the capacitive method (Corneometer CM 825). Int. J. Cosm. Sci. 25, 45-53(2003).

15) Ghadially, R.; Reed, J. T.; Elias, P. M. Stratum corneum structure and function correlates with phenotype in psoriasis. J. Invest. Dermatol. 107, 558-564(1996).

16) Takahashi, M. Skin care in atopic dermatitis: Characterizing dry skin in atopic dermatitis and utility of skin care. J. Jpn. Cosmetic Sci. Soc. 21, 50-55(1997).

17) Morse, N. L.; Clough, P. M. A meta-analysis of randomized, placebo-controlled clinical trials of Efamol evening primrose oil in atopic eczema. Where do we go from here in light of more recent discoveries? Curr. Pharm. Biotechnol. 7, 503-524(2006).

18) Kragballe, K. Dietary supplementation with a combination of n-3 and n- 6 fatty acids (super gamma-oil marine) improves psoriasis. Acta Derm. Venereol. 69, 265-268 (1989).

19) Yen, C. H.; Dai, Y. S.; Yang, Y. H.; Wang, L. C.; Lee, J. H.; Chiang, B. L. Linoleic acid metabolite levels and transepidermal water loss in children with atopic dermatitis. Ann. Allergy Asthma Immunol. 100, 66-73 (2008). 
20) Horrobin, D. F. Essential fatty acids in clinical dermatology. J. Am. Acad. Dermatol. 20, 1045-1053(1989).

21) Ziboh, V. A. The significance of polyunsaturated fatty acids in cutaneous biology. Lipids 31, S249-253 (1996).

22) Morse, P. F.; Horrobin D. F.; Manku M. S.; Stewart, J. C. M.; Allen, R.; Littlewood, S.; Wright, S.; Burton, J.;. Gould, D. J ; Holt, P. J.; Jansen, C. T.; Mattila, L.; Meigel, W.; Dettke, T. H.; Wexler, D.; Guenther, L.; Bordoni, A.; Patrizi, A. Meta-analysis of placebo-controlled studies of the efficacy of Epogam in the treatment of atopic eczema. Relationship between plasma essential fatty acid changes and clinical response. Br. J. Dermatol. 121, 75-90 (1989).

23) Nemoto-Hasebe, I.; Akiyama, M.; Nomura, T.; Sandilands, A.; McLean, W. H.; Shimizu, H. Clinical severity correlates with impaired barrier in filaggrin-related eczema. J. Invest. Dermatol. 129, 682-689(2009).

24) Jensen, J.; Fölster-Holst, R.; Baranowsky, A.; Schunck, M.; Winoto-Morbach, A.; Neumann, C.; Schütze S.; Proksch E. Impaired aphingomyelinase activity and epidermal differentiation in atopic dermatitis. J. Invest. Dermatol. 122, 1423-1431 (2004). 